\title{
Quasi-Random Graphs With Given Degree Sequences
}

\author{
Fan Chung,* Ron Graham $\uparrow$ \\ University of California, San Diego, La Jolla, California 92093; \\ e-mail: fan@ucsd.edu; graham@ucsd.edu
}

Received 3 May 2005; accepted 20 June 2006; received in final form 4 December 2006 Published online 14 August 2007 in Wiley InterScience (www.interscience.wiley.com). DOI 10.1002/rsa.20188

\begin{abstract}
It is now known that many properties of the objects in certain combinatorial structures are equivalent, in the sense that any object possessing any of the properties must of necessity possess them all. These properties, termed quasirandom, have been described for a variety of structures such as graphs, hypergraphs, tournaments, Boolean functions, and subsets of $\mathbf{Z}_{n}$, and most recently, sparse graphs. In this article, we extend these ideas to the more complex case of graphs which have a given degree sequence. (C) 2007 Wiley Periodicals, Inc. Random Struct. Alg., 32, 1-19, 2008
\end{abstract}

Keywords: quasi-random; graph; degree sequence

\section{INTRODUCTION}

During recent years there has been increasing interest in investigating the following phenomenon. For a given finite collection $C$ of "objects," suppose we have some probability distribution given on $C$. Typically, there are many properties which are satisfied by most (or almost all) of the objects in $C$ as seen in [4]. It turns out, however, that in many cases there is a large subclass $Q$ of these properties which are strongly correlated, in the sense that any object in $C$ which satisfies any of the properties in $Q$ must in fact necessarily satisfy all the properties in $Q$. Such properties are called "quasi-random." Specific cases where this behavior is investigated can be found in [14, 15, 21] (for graphs), [12,13, 16, 17] (for

Correspondence to: Fan Chung

*Supported by NSF Grants (DMS 0457215, ITR 0205061, ITR 0426858).

$\uparrow$ Supported by NSF Grant (CCR 0310991).

(C) 2007 Wiley Periodicals, Inc. 
hypergraphs), [19] (for tournaments), [18] (for sequences), [25] (for permutations), and [20] (for sparse graphs), for example.

In this article we will take $C$ to be the class $\mathcal{G}_{n}(\mathbf{d})$ of all graphs on $n$ vertices having some given degree sequence $\mathbf{d}$. This is rather different from the classical model of a random graph, in which all vertices have the same expected degree. Special cases of such graph families include the so-called power law graphs in which the number of vertices of degree $k$ is proportional to $k^{-\beta}$ for some positive real $\beta$. Such graphs arise in a variety of applications such as Web connectivity [2,5,6,9,24,26,28,29], communication networks [1,3], biological networks [22], collaboration graphs [27], etc.

In this article, we will introduce a class of quasi-random properties for $\mathcal{G}_{n}(\mathbf{d})$ and establish quantitative bounds on the strength of correlation between these properties. In particular, these results generalize and strengthen those in [20,21].

\section{NOTATION}

We will consider graphs $G=(V, E)$ where $V$ denotes the set of vertices of $G$ and $E$ denotes the set of edges of $G$. (For undefined graph theory terminology, see [33].) Our graphs will be undirected, having no loops or multiple edges. We will let $|V|$, the cardinality of $V$, be denoted by $n$.

If $\{x, y\} \in E$ is an edge of $G$, we say that $x$ and $y$ are adjacent, and write this as $x \sim y$. The neighborhood $\operatorname{nd}(x)$ of a vertex $x \in V$ is defined by

$$
\operatorname{nd}(x):=\{y \in V: y \sim x \text { in } G\} .
$$

For $x \in V$, the degree $d_{x}$ of $x$, denotes $|\operatorname{nd}(x)|$. The degree sequence $\mathbf{d}=\mathbf{d}_{G}$ of $G$ is given by

$$
\mathbf{d}=\left(d_{x}: x \in V\right),
$$

or equivalently, $\mathbf{d}$ can be viewed as a mapping $\mathbf{d}: V \rightarrow \mathbb{Z}^{+} \cup\{0\}$. For $X, Y \subseteq V$, define

$$
e(X, Y):=\mid\{(x, y): x \in X, y \in Y \text { and } x \sim y\} \mid .
$$

For $X \subseteq V$, define $\operatorname{vol}(X)$, the volume of $X$, by

$$
\operatorname{vol}(X)=\sum_{x \in X} d_{x}
$$

A walk $P=P_{t}(x, y)$ from $x$ to $y$ is a sequence $P=\left(x_{0}, x_{1}, \ldots, x_{t}\right)$, where $x_{0}=x, x_{t}=y$ and $x_{i} \sim x_{i+1}$ for $0 \leq i<t$. Such a walk is said to have length $t$. Here we do not require all $x_{i}$ 's to be distinct. If all $x_{i}$ 's are different, we say the walk is a path.

In this article, we consider graphs with every vertex having positive degree. The weight $w(P)$ of such a walk $P$ is defined to be

$$
w(P)=\prod_{0<i<t} \frac{1}{d_{x_{i}}}
$$

(thus, both endpoints are excluded in the product). If $P$ has length 1 (and therefore is an edge of $G)$, then $w(P)$ is defined to be 1 .

A circuit $C$ of length $t$ is a sequence of $t$ vertices $\left(x_{1}, x_{2}, \ldots, x_{t}\right)$ where $x_{i} \sim x_{i+1}, 1 \leq i<t$, and $x_{t} \sim x_{1}$. (We remark that in this definition, a circuit can be viewed as a rooted closed 
walk.) The weight $w(C)$ of such a circuit is defined by

$$
w(C)=\prod_{1 \leq i \leq t} \frac{1}{d_{x_{i}}} .
$$

The weighted adjacency matrix $M=M(G)$ is an $n \times n$ matrix with rows and columns indexed by $V$, defined by

$$
M(x, y)= \begin{cases}\frac{1}{\sqrt{d_{x} d_{y}}} & \text { if } x \sim y, \\ 0 & \text { otherwise. }\end{cases}
$$

Note that $M$ can be written as $M=I-\mathcal{L}$ where $I$ is the identity matrix and $\mathcal{L}$ denotes the (normalized) Laplacian (see [11]). The eigenvalues of $M$ are denoted by $\rho_{i}, 0 \leq i \leq n-1$, indexed so that

$$
1=\rho_{0} \geq\left|\rho_{1}\right| \geq\left|\rho_{2}\right| \geq \ldots \geq\left|\rho_{n-1}\right|
$$

using the Perron-Frobenius theorem. Note that $\rho_{0}=1$ has as its eigenvector $\left(\sqrt{d_{x}}\right)_{x \in V}$.

Finally, define for $X, Y \subseteq V$, and $t \geq 1$,

$$
e_{t}(X, Y)=\sum_{P \in P_{t}(X, Y)} w(P)
$$

where $P_{t}(X, Y)$ denotes the set of all walks of length $t$ between $x \in X$ and $y \in Y$. This is a weighted version of the number of walks of length $t$ between $X$ and $Y$. Note that $e_{1}(X, Y)=e(X, Y)$. In particular, $e_{1}(V, V)=\sum_{x} d_{x}=\operatorname{vol}(G)$. It is not difficult to check that for $t \geq 1$, we have $e_{t}(V, V)=\operatorname{vol}(G)$.

\section{THE QUASI-RANDOM PROPERTIES}

In this section we will state various properties that the $G \in \mathcal{G}_{n}(\mathbf{d})$ might satisfy. Each of these properties will depend on a parameter $\varepsilon$, which we will always assume to satisfy $0<\varepsilon<1$. The closer $\varepsilon$ is to 0 , the more the graph in question behaves like a random graph with respect to the property in question, that is, the more the value of the corresponding parameter is closer to its expected value for a random graph in $\mathcal{G}_{n}(\mathbf{d})$.

$\operatorname{DISC}(\varepsilon)$ :

For all $X, Y \subseteq V$,

$$
\left|e(X, Y)-\frac{\operatorname{vol}(X) \operatorname{vol}(Y)}{\operatorname{vol}(G)}\right| \leq \varepsilon \operatorname{vol}(G)
$$

$\operatorname{DISC}_{t}(\varepsilon)$ :

For all $X, Y \subseteq V$,

$$
\left|e_{t}(X, Y)-\frac{\operatorname{vol}(X) \operatorname{vol}(Y)}{\operatorname{vol}(G)}\right| \leq \varepsilon \operatorname{vol}(G) .
$$

Note that $\operatorname{DISC}_{1}(\varepsilon)$ is just $\operatorname{DISC}(\varepsilon)$.

EIG $(\varepsilon)$ :

With the matrix $M=M(G)=(M(x, y))_{x, y \in V}$ as defined in (1) and with eigenvalues satisfying

$$
1=\rho_{0} \geq\left|\rho_{1}\right| \geq\left|\rho_{2}\right| \geq \ldots \geq\left|\rho_{n-1}\right|,
$$


we have

$$
\left|\rho_{i}\right|<\varepsilon \quad \text { for all } i \geq 1 \text {. }
$$

$\operatorname{TRACE}_{2 t}(\varepsilon):$

The eigenvalues of $M$ satisfy

$$
\sum_{i \geq 1} \rho_{i}^{2 t} \leq \varepsilon
$$

$\operatorname{CIRCUIT}_{t}(\varepsilon)$ :

The weighted sum of the $t$-circuits $C_{t}$ in $G$ satisfies

$$
\left|\sum_{C_{t}: t-\text { circuit }} w\left(C_{t}\right)-1\right| \leq \varepsilon .
$$

We will prove the following implications in Section 4:

Theorem 1. For $t \geq 2$, the following implications hold.

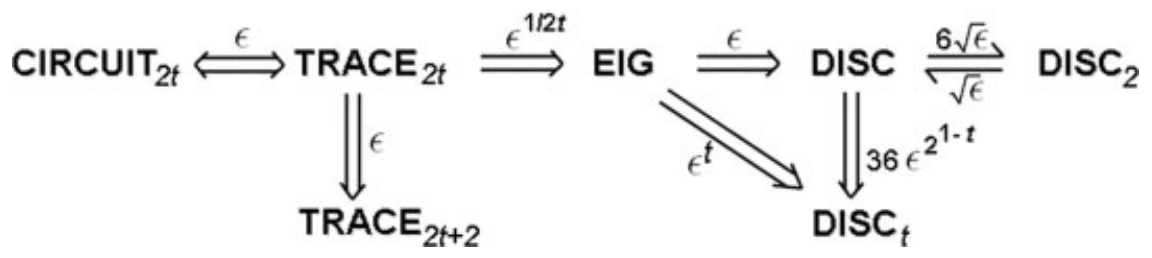

Fig. 1. Implications of several properties of $G_{n}(\mathbf{d})$.

Here the notation $A \stackrel{\delta}{\Rightarrow} B$ is shorthand for $A(\varepsilon) \Rightarrow B(\delta)$. We say $A$ implies $B$, denoted by $A \Rightarrow B$, if for every $\beta>0$, there exists $\alpha>0$ such that $A(\alpha) \Rightarrow B(\beta)$.

There are several one-way implications in the above Fig. 1. A natural question is which, if any, of the reverse directions hold for any of these implications. In Section 5, we will give counterexamples which show that EIG $\nRightarrow$ Trace $_{2 t}$ for any $t \geq 1$.

In Section 6, we introduce an additional property $\mathbf{U}_{t}$. Then we show that if a graph satisfies $\mathbf{U}_{t-1}$ for some $t \geq 2$, then DISC $\Rightarrow$ CIRCUIT $_{2 t}$. Using property $\mathbf{U}_{t}$, we will prove the following result.

Theorem 2. If $G$ satisfies $\mathbf{U}_{t-1}$ for some $t \geq 2$, then CIRCUIT $_{2 t}$, TRACE $_{2 t}$, EIG, DISC, $\mathrm{DISC}_{2}, \mathrm{DISC}_{t}$ are all equivalent.

\section{THE IMPLICATIONS}

Lemma 1. $\quad \operatorname{EIG}(\varepsilon) \Longrightarrow \operatorname{DISC}(\varepsilon)$.

Proof. For $S \subseteq V$, define

$$
f_{S}(x)= \begin{cases}\sqrt{d_{x}} & \text { if } x \in S \\ 0 & \text { otherwise. }\end{cases}
$$

Then, for $X, Y \subseteq V$,

$$
e(X, Y)=\left\langle f_{X}, M f_{Y}\right\rangle
$$

where $\langle f, g\rangle=\sum_{x \in V} f(x) g(x)$ denotes the usual inner product. 
Now, write

$$
f_{X}=\sum_{i} a_{i} \phi_{i}
$$

where the $\phi_{i}$ 's form an orthonormal basis of eigenvectors with

$$
\phi_{0}(v)=\sqrt{\frac{d_{v}}{\operatorname{vol}(G)}},
$$

for all $v \in V$. Hence,

$$
\begin{aligned}
a_{0} & =\left\langle f_{X}, \phi_{0}\right\rangle \\
& =\sum_{x \in X} \frac{d_{x}}{\sqrt{\operatorname{vol}(G)}} \\
& =\frac{\operatorname{vol}(X)}{\sqrt{\operatorname{vol}(G)}} .
\end{aligned}
$$

Similarly, we write

$$
f_{Y}=\sum_{i} b_{i} \phi_{i}
$$

Thus,

$$
\begin{aligned}
\left\langle f_{X}, M f_{Y}\right\rangle & =a_{0} b_{0}+\sum_{i \geq 1} \rho_{i} a_{i} b_{i} \\
& =\frac{\operatorname{vol}(X) \operatorname{vol}(Y)}{\operatorname{vol}(G)}+\sum_{i \geq 1} \rho_{i} a_{i} b_{i}
\end{aligned}
$$

Therefore,

$$
\begin{aligned}
\left|e(X, Y)-\frac{\operatorname{vol}(X) \operatorname{vol}(Y)}{\operatorname{vol}(G)}\right| & =\left|\sum_{i \geq 1} \rho_{i} a_{i} b_{i}\right| \\
& \leq \max _{i \geq 1}\left|\rho_{i}\right|\left(\sum_{i \geq 1}\left|a_{i}\right|^{2}\right)^{1 / 2}\left(\sum_{i \geq 1}\left|b_{i}\right|^{2}\right)^{1 / 2} \\
& \leq \varepsilon\left\|f_{X}\right\|\left\|f_{Y}\right\| \\
& =\varepsilon \sqrt{\operatorname{vol}(X) \operatorname{vol}(Y)} \\
& \leq \varepsilon \operatorname{vol}(G)
\end{aligned}
$$

by using EIG and the Cauchy-Schwarz inequality where $\|\cdot\|$ denotes the $L_{2}$-norm. Therefore, the proof is complete.

In a similar way, we prove

Lemma 2. $\quad E I G(\varepsilon) \Longrightarrow \operatorname{DISC}_{t}\left(\varepsilon^{t}\right)$ for any $t \geq 1$.

Proof. In this case we observe that for $X, Y \subseteq V$,

$$
e_{t}(X, Y)=\left\langle f_{X}, M^{t} f_{Y}\right\rangle
$$

Random Structures and Algorithms DOI 10.1002/rsa 
(using the notation of Lemma 1). Thus, writing

$$
f_{X}=\sum_{i} a_{i} \phi_{i}, f_{Y}=\sum_{i} b_{i} \phi_{i}
$$

we find

$$
\begin{aligned}
\left|\left\langle f_{X}, M^{t} f_{Y}\right\rangle-\frac{\operatorname{vol}(X) \operatorname{vol}(Y)}{\operatorname{vol}(G)}\right| & =\left|\left\langle f_{X}, M^{t} f_{Y}\right\rangle-\rho_{0}^{t} a_{0} b_{0}\right| \\
& \leq \max _{i \geq 1}\left|\rho_{i}\right|^{t} \sum_{i \geq 1}\left|a_{i} b_{i}\right| \\
& \leq \max _{i \geq 1}\left|\rho_{i}\right|^{t}\left\|f_{X}\right\|\left\|f_{Y}\right\| \\
& \leq \varepsilon^{t} \sqrt{\operatorname{vol}(X) \operatorname{vol}(Y)} \\
& \leq \varepsilon^{t} \operatorname{vol}(G)
\end{aligned}
$$

and Lemma 2 is proved.

Lemma 3. $\operatorname{CIRCUIT}_{2 t}(\varepsilon) \Longleftrightarrow \operatorname{TRACE}_{2 t}(\varepsilon)$.

Proof. Let $C_{2 t}^{*}(u)$ denote a rooted $2 t$-circuit with starting and ending point $u$. Then,

$$
M^{2 t}(u, u)=\sum_{C_{2 t}^{*}(u)} w\left(C_{2 t}^{*}(u)\right)
$$

Thus, the trace of the matrix $M^{2 t}$ can be expressed as:

$$
\begin{aligned}
\operatorname{Tr}\left(M^{2 t}\right) & =\sum_{u} \sum_{C_{2 t}^{*}(u)} w\left(C_{2 t}^{*}(u)\right) \\
& =\sum_{C_{2 t}} w\left(C_{2 t}\right)
\end{aligned}
$$

On the other hand, the same trace can be evaluated using eigenvalues:

$$
\begin{aligned}
\operatorname{Tr}\left(M^{2 t}\right) & =\sum_{i} \rho_{i}^{2 t} \\
& =1+\sum_{i \geq 1} \rho_{i}^{2 t} .
\end{aligned}
$$

Thus, we have

$$
\begin{aligned}
\left|\sum_{C_{2 t}} w\left(C_{2 t}\right)-1\right| & =\left|\operatorname{Tr}\left(M^{2 t}\right)-1\right| \\
& =\sum_{i \geq 1} \rho_{i}^{2 t}
\end{aligned}
$$

and Lemma 3 is proved. 
Lemma 4. $\operatorname{TRACE}_{2 t}(\varepsilon) \Longrightarrow \operatorname{EIG}\left(\varepsilon^{1 / 2 t}\right)$, for any $t \geq 1$.

Proof. By hypothesis, we have

$$
\left|\sum_{i} \rho_{i}^{2 t}-1\right|=\sum_{i \geq 1} \rho_{i}^{2 t} \leq \varepsilon .
$$

Therefore,

$$
\max _{i \geq 1}\left|\rho_{i}\right| \leq \varepsilon^{1 / 2 t}
$$

Lemma 5. $\operatorname{TRACE}_{2 t}(\varepsilon) \Longrightarrow \operatorname{TRACE}_{2 t+2}(\varepsilon)$.

Proof. Since $\left|\rho_{i}\right| \leq 1$ for all $i$, we have

$$
\sum_{i \geq 1} \rho_{i}^{2 t+2} \leq \sum_{i \geq 1} \rho_{i}^{2 t} \leq \varepsilon
$$

by hypothesis.

Lemma 6. For $t \geq 1, \operatorname{DISC}_{2 t}(\varepsilon) \Longrightarrow \operatorname{DISC}_{t}(\sqrt{\varepsilon})$.

Proof. For $X \subseteq V$,

$$
\begin{aligned}
e_{2 t}(X, X) & =\sum_{x, x^{\prime} \in X} \sum_{y} \frac{e_{t}(x, y) e_{t}\left(y, x^{\prime}\right)}{d_{y}} \\
& =\sum_{y} \frac{e_{t}(y, X)^{2}}{d_{y}} .
\end{aligned}
$$

By applying $\operatorname{DISC}_{2 t}(\varepsilon)$ to $e_{2 t}(X, X)$, we have

$$
\sum_{y} \frac{e_{t}(y, X)^{2}}{d_{y}} \leq \frac{\operatorname{vol}(X)^{2}}{\operatorname{vol}(G)}+\varepsilon \operatorname{vol}(G) .
$$

Note that

$$
\sum_{y} e_{t}(y, X)=e_{t}(V, X)=\sum_{x \in V} d_{x}=\operatorname{vol}(X)
$$

Therefore,

$$
\begin{aligned}
& \sum_{y}\left(e_{t}(y, X)-\frac{d_{y} \operatorname{vol}(X)}{\operatorname{vol}(G)}\right)^{2} \frac{1}{d_{y}} \\
= & \sum_{y} \frac{e_{t}(y, X)^{2}}{d_{y}}-2 e_{t}(V, X) \frac{\operatorname{vol}(X)}{\operatorname{vol}(G)}+\frac{\operatorname{vol}(X)^{2}}{\operatorname{vol}(G)} \\
= & \sum_{y} \frac{e_{t}(y, X)^{2}}{d_{y}}-\frac{\operatorname{vol}(X)^{2}}{\operatorname{vol}(G)} \\
\leq & \varepsilon \operatorname{vol}(G) .
\end{aligned}
$$


by (2) and $\operatorname{DISC}_{2 t}(\varepsilon)$. But

$$
\begin{aligned}
& \sum_{y}\left(e_{t}(y, X)-\frac{d_{y} \operatorname{vol}(X)}{\operatorname{vol}(G)}\right)^{2} \frac{1}{d_{y}} \\
\geq & \sum_{y \in Y}\left(e_{t}(y, X)-\frac{d_{y} \operatorname{vol}(X)}{\operatorname{vol}(G)}\right)^{2} \frac{1}{d_{y}} \\
\geq & \left(\sum_{y \in Y}\left(e_{t}(y, X)-d_{y} \frac{\operatorname{vol}(X)}{\operatorname{vol}(G)}\right)\right)^{2} \frac{1}{\sum_{y \in Y} d_{y}} \\
= & \left(e_{t}(Y, X)-\frac{\operatorname{vol}(Y) \operatorname{vol}(X)}{\operatorname{vol}(G)}\right)^{2} / \operatorname{vol}(Y)
\end{aligned}
$$

by applying the Cauchy-Schwarz inequality. Thus,

$$
\begin{aligned}
\left|e_{t}(Y, X)-\frac{\operatorname{vol}(Y) \operatorname{vol}(X)}{\operatorname{vol}(G)}\right| & \leq \sqrt{\varepsilon \operatorname{vol}(Y) \operatorname{vol}(G)} \\
& \leq \sqrt{\varepsilon} \operatorname{vol}(G) .
\end{aligned}
$$

This is exactly $\operatorname{DISC}_{t}(\sqrt{\varepsilon})$.

Lemma 7. For any $t \geq 1, \operatorname{DISC}_{t}(\varepsilon) \Longrightarrow \operatorname{DISC}_{t+1}(6 \sqrt{\varepsilon})$.

Proof. For $X, Y \subseteq V$,

$$
\left|e_{t}(X, Y)-\frac{\operatorname{vol}(X) \operatorname{vol}(Y)}{\operatorname{vol}(G)}\right| \leq \varepsilon \operatorname{vol}(G)
$$

Consider

$$
e_{t+1}(X, Y)=\sum_{v} \frac{e(X, v) e_{t}(v, Y)}{d_{v}}
$$

Define

$$
S_{1}:=\left\{z \in V: e_{t}(z, Y)>\frac{d_{z}}{\operatorname{vol}(G)}(\operatorname{vol}(Y)+\sqrt{\varepsilon} \operatorname{vol}(G))\right\} .
$$

Thus,

$$
\sum_{z \in S_{1}} e_{t}(z, Y)=e_{t}\left(S_{1}, Y\right)>\frac{\operatorname{vol}\left(S_{1}\right) \operatorname{vol}(Y)}{\operatorname{vol}(G)}+\sqrt{\varepsilon} \operatorname{vol}\left(S_{1}\right) .
$$

Hence, by (3) applied to $X=S_{1}$ and $Y$,

$$
\operatorname{vol}\left(S_{1}\right)<\sqrt{\varepsilon} \operatorname{vol}(G) .
$$

In the same way, if we define $S_{2}:=\left\{z \in V: e_{t}(z, Y)<\frac{d_{z}}{\operatorname{vol}(G)}(\operatorname{vol}(Y)-\sqrt{\varepsilon} \operatorname{vol}(G))\right\}$, then

$$
\operatorname{vol}\left(S_{2}\right)<\sqrt{\varepsilon} \operatorname{vol}(G)
$$

Now,

$$
e_{t+1}(X, Y)=\left(\sum_{v \notin S_{1} \cup S_{2}}+\sum_{v \in S_{1} \cup S_{2}}\right) \frac{e(X, v) e_{t}(v, Y)}{d_{v}} .
$$

Random Structures and Algorithms DOI 10.1002/rsa 
For the first sum, we have

$$
\begin{aligned}
\sum_{v \notin S_{1} \cup S_{2}} \frac{e(X, v) e_{t}(v, Y)}{d_{v}} & \leq \sum_{v \notin S_{1} \cup S_{2}} \frac{e(X, v)}{d_{v}} \frac{d_{v}}{\operatorname{vol}(G)}(\operatorname{vol}(Y)+\sqrt{\varepsilon} \operatorname{vol}(G)) \\
& \leq \sum_{v} \frac{e(X, v)}{\operatorname{vol}(G)}(\operatorname{vol}(Y)+\sqrt{\varepsilon} \operatorname{vol}(G)) \\
& \leq \frac{\operatorname{vol}(X) \operatorname{vol}(Y)}{\operatorname{vol}(G)}+\sqrt{\varepsilon} \operatorname{vol}(G)
\end{aligned}
$$

and

$$
\begin{aligned}
\sum_{v \notin S_{1} \cup S_{2}} \frac{e(X, v) e_{t}(v, Y)}{d_{v}} & \geq \sum_{v \notin S_{1} \cup S_{2}} \frac{e(X, v)}{d_{v}} \frac{d_{v}}{\operatorname{vol}(G)}(\operatorname{vol}(Y)-\sqrt{\varepsilon} \operatorname{vol}(G)) \\
& \geq \sum_{v \notin S_{1} \cup S_{2}} \frac{e(X, v)}{\operatorname{vol}(G)}(\operatorname{vol}(Y)-\sqrt{\varepsilon} \operatorname{vol}(G)) \\
& \geq \frac{\left(\operatorname{vol}(X)-\operatorname{vol}\left(S_{1}\right)-\operatorname{vol}\left(S_{2}\right)\right)}{\operatorname{vol}(G)}(\operatorname{vol}(Y)-\sqrt{\varepsilon} \operatorname{vol}(G)) \\
& \geq \frac{(\operatorname{vol}(X)-2 \sqrt{\varepsilon} \operatorname{vol}(G))}{\operatorname{vol}(G)}(\operatorname{vol}(Y)-\sqrt{\varepsilon} \operatorname{vol}(G)) \\
& \geq \frac{\operatorname{vol}(X) \operatorname{vol}(Y)}{\operatorname{vol}(G)}-3 \sqrt{\varepsilon} \operatorname{vol}(G),
\end{aligned}
$$

by using (4) and (5). Thus,

$$
\left|\sum_{v \notin S_{1} \cup S_{2}} \frac{e(X, v) e_{t}(v, Y)}{d_{v}}-\frac{\operatorname{vol}(X) \operatorname{vol}(Y)}{\operatorname{vol}(G)}\right| \leq 3 \sqrt{\varepsilon} \operatorname{vol}(G) .
$$

For the second sum, we have

$$
\begin{aligned}
\sum_{v \in S_{1} \cup S_{2}} \frac{e(X, v) e_{t}(v, Y)}{d_{v}} & \leq \sum_{v \in S_{1} \cup S_{2}} \frac{d_{v} e_{t}(v, Y)}{d_{v}} \\
& =e_{t}\left(S_{1} \cup S_{2}, Y\right) \\
& \leq \frac{\left(\operatorname{vol}\left(S_{1}\right)+\operatorname{vol}\left(S_{2}\right)\right) \operatorname{vol}(Y)}{\operatorname{vol}(G)}+\varepsilon \operatorname{vol}(G) \quad \text { by } \operatorname{DISC}_{t}(\varepsilon) \\
& \leq \frac{2 \sqrt{\varepsilon} \operatorname{vol}(G) \operatorname{vol}(Y)}{\operatorname{vol}(G)}+\varepsilon \operatorname{vol}(G) \\
& =2 \sqrt{\varepsilon} \operatorname{vol}(Y)+\varepsilon \operatorname{vol}(G) \\
& \leq 3 \sqrt{\varepsilon} \operatorname{vol}(G)
\end{aligned}
$$

Putting these two estimates together, we obtain

$$
\left|e_{t+1}(X, Y)-\frac{\operatorname{vol}(X) \operatorname{vol}(Y)}{\operatorname{vol}(G)}\right| \leq 6 \sqrt{\varepsilon} \operatorname{vol}(G)
$$

which is $\operatorname{DISC}_{t+1}(6 \sqrt{\varepsilon})$.

Random Structures and Algorithms DOI 10.1002/rsa 
Lemma 8. For any integers $s$ and $t, D I S C_{s}$ and $D I S C_{t}$ are related as follows:

i. If $s<t$, then $\operatorname{DISC}_{s}(\varepsilon) \Longrightarrow \operatorname{DISC}_{t}\left(36 \varepsilon^{1 / 2^{t-s}}\right)$. As a special case, $\operatorname{DISC}(\varepsilon) \Longrightarrow \operatorname{DISC}_{t}\left(36 \varepsilon^{1 / 2^{t-1}}\right)$.

ii. If $t<s \leq 2^{k}$ for some $k$, then $\operatorname{DISC}_{s}(\varepsilon) \Longrightarrow \operatorname{DISC}_{t}\left(36^{1 / 2^{k}} \varepsilon^{1 / 2^{k^{k}+k-s}}\right)$.

Proof. (i) follows from Lemma 7, i.e.,

$$
\begin{aligned}
\operatorname{DISC}_{s}(\varepsilon) & \Rightarrow \operatorname{DISC}_{s}(36 \varepsilon) \\
& \Rightarrow \operatorname{DISC}_{s+1}\left(36 \varepsilon^{1 / 2}\right) \\
& \Rightarrow \ldots \\
& \Rightarrow \operatorname{DISC}_{t}\left(36 \varepsilon^{1 / 2^{t-s}}\right)
\end{aligned}
$$

To prove (ii), we have, from (i) that

$$
\operatorname{DISC}_{s}(\varepsilon) \Rightarrow \operatorname{DISC}_{2^{k_{t}}}\left(36 \varepsilon^{1 / 2^{2^{k} t-s}}\right)
$$

Now apply Lemma $6 k$ times to get the desired implication.

By combining Lemmas 1 to 8, we have proved all the implications in Theorem 1.

\section{SEPARATION OF PROPERTIES}

In this section, we give an example showing that at least one of the implications in Theorem 1 cannot be reversed. Whether this is true of the others is not known at this point.

Fact 1. For any $t \geq 1$,

$$
\operatorname{EIG}(\varepsilon) \not \operatorname{TRACE}_{2 t}(\delta)
$$

for any $\delta=\delta(\varepsilon)$.

Proof. Choose $t \geq 1$ and let $G=G(n)$ be a random regular graph with $n$ vertices and vertex degree $n^{1 / t}$. Thus, $M=M(G)$ has

$$
M(u, v)= \begin{cases}1 / n^{1 / t} & \text { if } u \sim v, \\ 0 & \text { otherwise }\end{cases}
$$

It was shown in [23] that the eigenvalue distribution of $M(G)$ for a random graph $G$ with a given expected degree distribution satisfies the semi-circle law if the minimum degree is greater than a power of $\log n$. As a consequence, if $1=\rho_{0} \geq\left|\rho_{1}\right| \geq\left|\rho_{2}\right| \geq \ldots \geq\left|\rho_{n-1}\right|$ are the eigenvalues of $M$, then

1. $\rho_{1}=(1+o(1)) 2 / n^{1 / 2 t}$,

2. If $N(x)$ denotes the number of $\rho_{i}$ with $\rho_{i} \leq 2 x / n^{1 / 2 t}$, then

$$
\frac{N(x)}{n}=(1+o(1)) \frac{2}{\pi} \int_{-1}^{x} \sqrt{1-u^{2}} d u .
$$


In particular, for $x=1 / 2$, we have

$$
\frac{N(1 / 2)}{n}=(1+o(1))\left(\frac{2}{3}+\frac{\sqrt{3}}{4 \pi}\right) \approx 0.8045 \ldots
$$

Thus,

$$
\sum_{i \geq 1} \rho_{i}^{2 t} \geq 2(n-N(1 / 2))\left(\frac{1}{n^{1 / 2 t}}\right)^{2 t} \geq 0.391 .
$$

Hence, for any $\varepsilon>0, G$ satisfies $\operatorname{EIG}(\varepsilon)$, provided $n \geq n_{0}$, but $G$ does not satisfy $\operatorname{TRACE}_{2 t}(0.39)$.

It would be interesting to know if some of the other possible implications hold. For example, does DISC $\Rightarrow$ EIG?

Recently, Bilu and Linial [7] proved the following partial implication for regular graphs:

For a $d$-regular graph $G$ on $n$ vertices, if for all $X, Y \subset V$,

$$
\left|e(X, Y)-\frac{d}{n}\right| X|| Y|| \leq \alpha d \sqrt{|X||Y|},
$$

then $\left|\rho_{1}\right|=O(\alpha(\log (1 / \alpha)+1))$.

The above property in (6) was introduced by Thomason [31] in the context of what he called $(p, \alpha)$-jumbled graphs. Of course, this property is quite a bit stronger than DISC. Properties of random graphs based on this concept (without the equivalence relations) are often referred as the pseudo-random properties. The reader is referred to [30,32] for discussions on pseudo-random graphs.

Butler [10] combines the methods in [7] and [8] to prove the following:

For a graph $G$ with no isolated vertices, if for all $X, Y \subset V$,

$$
\left|e_{t}(X, Y)-\frac{\operatorname{vol}(X) \operatorname{vol}(Y)}{\operatorname{vol}(G)}\right| \leq \alpha \sqrt{\operatorname{vol}(X) \operatorname{vol}(Y)}
$$

then $\left|\rho_{1}\right|^{t} \leq 18 \alpha\left(1-\frac{5}{2} \log \alpha\right)$.

For $t=1$, this is the best possible (up to a constant) by considering a class of regular graphs constructed by Bollobás and Nikiforov [8]. In their example, the graphs have $\alpha=$ $C n^{-1 / 6}$ and $\left|\rho_{1}\right| \geq c \alpha \log n$ for some constants $c$ and $C$.

\section{REVERSING THE IMPLICATIONS}

It is clear from the examples in the preceding section that in order to establish some of the reverse implications, e.g., DISC $\Rightarrow$ CIRCUIT $_{2 t}$, we will have to make further assumptions for the $G \in \mathcal{G}_{n}(\mathbf{d})$. One such condition is the following:

For $t \geq 1$, a graph satisfies $U_{t}(C)$ if for all $x, y \in V, e_{t}(x, y) \leq C \frac{d_{x} d_{y}}{\operatorname{vol}(G)}$.

We will think of $C$ as a large positive real. We note that for $t=1$ and for $G$ with minimum degree $\alpha n$, the property $U_{1}(C)$ is automatically satisfied for $C \geq 1 / \alpha^{2}$.

Note that for a $d$-regular graph, $U_{t}$ implies that $n \leq C d^{t}$ or, equivalently, the volume of the graph is of order at least $n^{1+1 / t}$. 
Lemma 9. For any $t \geq 1$,

$$
U_{t}(C) \Longrightarrow U_{t+1}(C)
$$

Proof. Observe that

$$
\begin{aligned}
e_{t+1}(x, y) & =\sum_{z} \frac{e(x, z) e_{t}(z, y)}{d_{z}} \\
& \leq \sum_{z} \frac{e(x, z)}{d_{z}} \cdot C \frac{d_{z} d_{y}}{\operatorname{vol}(G)} \\
& =C \frac{d_{y}}{\operatorname{vol}(G)} \sum_{z} e(x, z) \\
& =C \frac{d_{x} d_{y}}{\operatorname{vol}(G)} .
\end{aligned}
$$

The lemma is proved.

Theorem 3. If $G$ satisfies $U_{t-1}(C)$ for some $t \geq 2$, then

$$
\operatorname{DISC}(\varepsilon) \Longrightarrow \operatorname{CIRCUIT}_{2 t}(\eta)
$$

where $\eta=2 C^{\prime} C^{2} \varepsilon / \delta+2 C^{2}\left(C^{\prime}+1\right)^{2} \delta+20 \sqrt{\delta}+12 \delta+16 C^{4} \delta^{3 / 2}+8 C^{\prime 2} \delta^{3 / 2}$, with $C^{\prime}=$ $\left\lceil C / \delta^{1 / 4}\right\rceil$, and $\delta=\max \left\{\sqrt{\varepsilon}, 36 \varepsilon^{1 / 2^{t-2}}\right\}$. (Note that $\eta \rightarrow 0$ as $\varepsilon \rightarrow 0$.)

Proof. We are going to consider the sum

$$
\sum_{u, v \in V} \frac{1}{d_{u} d_{v}}\left(e_{t}(u, v)-\frac{d_{u} d_{v}}{\operatorname{vol}(G)}\right)^{2}
$$

where, as usual, $V=V(G)$.

Since $G$ satisfies $\operatorname{DISC}(\varepsilon)$ by hypothesis, then by Lemma $8, G$ also satisfies $\operatorname{DISC}_{t-1}(\delta)$ where $\delta \geq 36 \varepsilon^{1 / 2^{t-2}}$, i.e.,

$$
\left|e_{t-1}(X, Y)-\frac{\operatorname{vol}(X) \operatorname{vol}(Y)}{\operatorname{vol}(G)}\right| \leq \delta \operatorname{vol}(G)
$$

for all $X, Y \subseteq V$.

We here choose $\delta=\max \left\{\sqrt{\varepsilon}, 36 \varepsilon^{1 / 2^{t-2}}\right\}$. For a fixed vertex $u$, we partition the vertex set $V$ into the sets $W_{i}=W_{i}(u), 0 \leq i<C^{\prime}$, as follows. (To simplify the notation, we use $W_{i}$ instead of $W_{i}(u)$ below.)

$$
\begin{aligned}
& W_{0}=\left\{v: 0 \leq e_{t-1}(u, v)<\delta^{1 / 4} \frac{d_{u} d_{v}}{\operatorname{vol}(G)}\right\}, \\
& W_{1}=\left\{v: \delta^{1 / 4} \frac{d_{u} d_{v}}{\operatorname{vol}(G)} \leq e_{t-1}(u, v)<2 \delta^{1 / 4} \frac{d_{u} d_{v}}{\operatorname{vol}(G)}\right\}, \\
& W_{2}=\left\{v: 2 \delta^{1 / 4} \frac{d_{u} d_{v}}{\operatorname{vol}(G)} \leq e_{t-1}(u, v)<3 \delta^{1 / 4} \frac{d_{u} d_{v}}{\operatorname{vol}(G)}\right\},
\end{aligned}
$$


and, in general,

$$
W_{i}=\left\{v: i \delta^{1 / 4} \frac{d_{u} d_{v}}{\operatorname{vol}(G)} \leq e_{t-1}(u, v)<(i+1) \delta^{1 / 4} \frac{d_{u} d_{v}}{\operatorname{vol}(G)}\right\}
$$

for $0 \leq i<C^{\prime}=\left\lceil C / \delta^{1 / 4}\right\rceil$. Since $e_{t-1}(u, v) \leq C d_{u} d_{v} / \operatorname{vol}(G)$ by $\mathrm{U}_{t-1}(C)$, the $W_{i}$ form a partition of $V$.

Since

$$
W_{i} \subseteq\left\{v:\left|e_{t-1}(u, v)-i \delta^{1 / 4} \frac{d_{u} d_{v}}{\operatorname{vol}(G)}\right|<\delta^{1 / 4} \frac{d_{u} d_{v}}{\operatorname{vol}(G)}\right\}
$$

then

$$
\begin{aligned}
&\left|\sum_{v \in W_{i}}\left(e_{t-1}(u, v)-i \delta^{1 / 4} \frac{d_{u} d_{v}}{\operatorname{vol}(G)}\right)\right|<\sum_{v \in W_{i}} \delta^{1 / 4} \frac{d_{u} d_{v}}{\operatorname{vol}(G)} \\
& \text { and }\left|e_{t-1}\left(u, W_{i}\right)-i \delta^{1 / 4} \frac{d_{u} \operatorname{vol}\left(W_{i}\right)}{\operatorname{vol}(G)}\right|<\delta^{1 / 4} \frac{d_{u} \operatorname{vol}\left(W_{i}\right)}{\operatorname{vol}(G)} .
\end{aligned}
$$

Since $\sum_{i} e_{t-1}\left(u, W_{i}\right)=e_{t-1}(u, V)=d_{u}$, then

$$
\begin{aligned}
& \left|\sum_{i} i \delta^{1 / 4} \frac{d_{u} \operatorname{vol}\left(W_{i}\right)}{\operatorname{vol}(G)}-d_{u}\right| \\
= & \left|\sum_{i} i \delta^{1 / 4} \frac{d_{u} \operatorname{vol}\left(W_{i}\right)}{\operatorname{vol}(G)}-\sum_{i} e_{t-1}\left(u, W_{i}\right)\right| \\
\leq & \delta^{1 / 4} \sum_{i} \frac{d_{u} \operatorname{vol}\left(W_{i}\right)}{\operatorname{vol}(G)} \\
= & \delta^{1 / 4} d_{u} .
\end{aligned}
$$

Now, for each $i$, if $\operatorname{vol}\left(W_{i}\right) \geq \sqrt{\delta} \operatorname{vol}(G)$, then define $X_{i}=X_{i}(u)$ and $X_{i}^{\prime}=X_{i}^{\prime}(u)$ as follows:

$$
\begin{aligned}
& X_{i}=\left\{v: e\left(v, W_{i}\right)>\frac{d_{v} \operatorname{vol}\left(W_{i}\right)}{\operatorname{vol}(G)}(1+\sqrt{\delta})\right\}, \\
& X_{i}^{\prime}=\left\{v: e\left(v, W_{i}\right)<\frac{d_{v} \operatorname{vol}\left(W_{i}\right)}{\operatorname{vol}(G)}(1-\sqrt{\delta})\right\} .
\end{aligned}
$$

If $\operatorname{vol}\left(W_{i}\right)<\sqrt{\delta} \operatorname{vol}(G)$ then define $X_{i}=X_{i}^{\prime}=\emptyset$. Also define

$$
W_{u}^{*}=\bigcup\left\{W_{i}: \operatorname{vol}\left(W_{i}\right)<\sqrt{\delta} \operatorname{vol}(G)\right\} .
$$

Thus,

$$
\operatorname{vol}\left(W_{u}^{*}\right) \leq C^{\prime} \sqrt{\delta} \operatorname{vol}(G)
$$

since there are just $C^{\prime}$ possible values of $i$.

$\operatorname{By} \operatorname{DISC}(\varepsilon)$, we have

$$
\left|e\left(W_{i}, X_{i}\right)-\frac{\operatorname{vol}\left(W_{i}\right) \operatorname{vol}\left(X_{i}\right)}{\operatorname{vol}(G)}\right| \leq \varepsilon \operatorname{vol}(G),
$$


but from the definition of $X_{i}$, we have

$$
\begin{aligned}
\left|e\left(W_{i}, X_{i}\right)-\frac{\operatorname{vol}\left(W_{i}\right) \operatorname{vol}\left(X_{i}\right)}{\operatorname{vol}(G)}\right| & \geq \sqrt{\delta} \frac{\operatorname{vol}\left(X_{i}\right) \operatorname{vol}\left(W_{i}\right)}{\operatorname{vol}(G)} \\
& \geq \sqrt{\delta} \frac{\sqrt{\delta} \operatorname{vol}(G) \operatorname{vol}\left(X_{i}\right)}{\operatorname{vol}(G)} \\
& =\delta \operatorname{vol}\left(X_{i}\right) .
\end{aligned}
$$

Therefore,

$$
\operatorname{vol}\left(X_{i}\right) \leq \varepsilon / \delta \operatorname{vol}(G) .
$$

A similar argument shows that

$$
\operatorname{vol}\left(X_{i}^{\prime}\right) \leq \varepsilon / \delta \operatorname{vol}(G)
$$

as well. Consequently, for each $u$, we consider

$$
X_{u}:=\bigcup\left\{X_{i} \cup X_{i}^{\prime}: W_{i} \nsubseteq W_{u}^{*}\right\}
$$

and we have

$$
\operatorname{vol}\left(X_{u}\right) \leq 2 C^{\prime} \varepsilon / \delta \operatorname{vol}(G) .
$$

For $v \notin X_{u}$, we have, from the definition of $X_{u}$,

$$
\begin{aligned}
e\left(W_{u}^{*}, v\right) & =d_{v}-\sum_{W_{i} \notin W_{u}^{*}} e\left(W_{i}, v\right) \\
& \leq d_{v}-\sum_{W_{i} \notin W_{u}^{*}}(1-\sqrt{\delta}) \frac{d_{v} \operatorname{vol}\left(W_{i}\right)}{\operatorname{vol}(G)} \\
& =d_{v}-(1-\sqrt{\delta}) \frac{d_{v}\left(\operatorname{vol}(G)-\operatorname{vol}\left(W_{u}^{*}\right)\right)}{\operatorname{vol}(G)} \\
& =\sqrt{\delta} d_{v}+(1-\sqrt{\delta}) \frac{\operatorname{vol}\left(W_{u}^{*}\right)}{\operatorname{vol}(G)} d_{v} \\
& \leq \sqrt{\delta} d_{v}+(1-\sqrt{\delta}) C^{\prime} \frac{\sqrt{\delta} \operatorname{vol}(G)}{\operatorname{vol}(G)} d_{v} \\
& \leq\left(C^{\prime}+1\right) \sqrt{\delta} d_{v} .
\end{aligned}
$$

We now begin considering the sum,

$$
\sum_{u} \sum_{v} \frac{1}{d_{u} d_{v}}\left(e_{t}(u, v)-\frac{d_{u} d_{v}}{\operatorname{vol}(G)}\right)^{2}=\sum_{u}\left(\sum_{v \in X_{u}}+\sum_{v \notin X_{u}}\right) \frac{1}{d_{u} d_{v}}\left(e_{t}(u, v)-\frac{d_{u} d_{v}}{\operatorname{vol}(G)}\right)^{2} .
$$

For the first sum, we use property $\mathbf{U}_{t}(C)$ and Lemma 9 to obtain the following estimate:

$$
\begin{aligned}
\sum_{u} \sum_{v \in X_{u}} \frac{1}{d_{u} d_{v}}\left(e_{t}(u, v)-\frac{d_{u} d_{v}}{\operatorname{vol}(G)}\right)^{2} & \leq \sum_{u} \sum_{v \in X_{u}} \frac{1}{d_{u} d_{v}} C^{2}\left(\frac{d_{u} d_{v}}{\operatorname{vol}(G)}\right)^{2} \\
& =C^{2} \frac{\operatorname{vol}\left(X_{u}\right)}{\operatorname{vol}(G)} \\
& \leq 2 C^{\prime} C^{2} \varepsilon / \delta
\end{aligned}
$$


by (9). For the second sum we have

$$
\begin{aligned}
& \sum_{u} \sum_{v \notin X_{u}} \frac{1}{d_{u} d_{v}}\left(e_{t}(u, v)-\frac{d_{u} d_{v}}{\operatorname{vol}(G)}\right)^{2} \\
= & \sum_{u} \sum_{v \notin X_{u}} \frac{1}{d_{u} d_{v}}\left(\sum_{z} \frac{e_{t-1}(u, z) e(z, v)}{d_{z}}-\frac{d_{u} d_{v}}{\operatorname{vol}(G)}\right)^{2} \\
= & \sum_{u} \sum_{v \notin X_{u}} \frac{1}{d_{u} d_{v}}\left(\left(\sum_{z \in W_{u}^{*}}+\sum_{z \notin W_{u}^{*}}\right) \frac{e_{t-1}(u, z) e(z, v)}{d_{z}}-\frac{d_{u} d_{v}}{\operatorname{vol}(G)}\right)^{2} \\
\leq & \sum_{u} \sum_{v \notin X_{u}} \frac{2}{d_{u} d_{v}}\left(\left(\sum_{z \in W_{u}^{*}} \frac{e_{t-1}(u, z) e(z, v)}{d_{z}}\right)^{2}+\left(\sum_{z \notin W_{u}^{*}} \frac{e_{t-1}(u, z) e(z, v)}{d_{z}}-\frac{d_{u} d_{v}}{\operatorname{vol}(G)}\right)^{2}\right) .
\end{aligned}
$$

For the first sum just above (without a factor of 2), we have

$$
\begin{aligned}
& \sum_{u} \sum_{v \notin X_{u}} \frac{1}{d_{u} d_{v}}\left(\sum_{z \in W_{u}^{*}} \frac{e_{t-1}(u, z) e(z, v)}{d_{z}}\right)^{2} \\
\leq & \sum_{u} \sum_{v \notin X_{u}} \frac{1}{d_{u} d_{v}}\left(\sum_{z \in W_{u}^{*}} \frac{C d_{u} d_{z} e(z, v)}{d_{z} \operatorname{vol}(G)}\right)^{2} \quad \text { by } \mathrm{U}_{t-1}(C), \\
= & \sum_{u} \sum_{v \notin X_{u}} \frac{C^{2} d_{u}^{2}\left(e\left(W_{u}^{*}, v\right)\right)^{2}}{d_{u} d_{v} \operatorname{vol}(G)^{2}} \\
\leq & \sum_{u} \sum_{v \notin X_{u}} \frac{C^{2}\left(C^{\prime}+1\right)^{2} \delta d_{u} d_{v}}{\operatorname{vol}(G)^{2}} \text { by }(10) \\
\leq & C^{2}\left(C^{\prime}+1\right)^{2} \delta .
\end{aligned}
$$

For the second sum above, we have

$$
\begin{aligned}
& \sum_{u} \sum_{v \notin X_{u}} \frac{1}{d_{u} d_{v}}\left(\sum_{z \notin W_{u}^{*}} \frac{e_{t-1}(u, z) e(z, v)}{d_{z}}-\frac{d_{u} d_{v}}{\operatorname{vol}(G)}\right)^{2} \\
= & \sum_{u} \sum_{v \notin X_{u}} \frac{1}{d_{u} d_{v}}\left(\sum_{W_{i} \notin W_{u}^{*}} \sum_{z \in W_{i}} \frac{e_{t-1}(u, z) e(z, v)}{d_{z}}-\frac{d_{u} d_{v}}{\operatorname{vol}(G)}\right)^{2} \\
\leq & \sum_{u} \sum_{v \notin X_{u}} \frac{2}{d_{u} d_{v}}\left(\left(\sum_{W_{i} \notin W_{u}^{*}} \sum_{z \in W_{i}} \frac{i \delta^{1 / 4} d_{u} d_{z} e(z, v)}{d_{z} \operatorname{vol}(G)}-\frac{d_{u} d_{v}}{\operatorname{vol}(G)}\right)^{2}\right. \\
& \left.+\left(\sum_{W_{i} \notin W_{u}^{*}} \sum_{z \in W_{i}} \frac{\delta^{1 / 4} d_{u} d_{z} e(z, v)}{d_{z} \operatorname{vol}(G)}\right)^{2}\right)
\end{aligned}
$$

since $|A-a| \leq b \Rightarrow(A-B)^{2} \leq 2\left((a-B)^{2}+b^{2}\right)$ and inequalities in (7). 
For the second sum we have

$$
\begin{aligned}
& \sum_{u} \sum_{v \notin X_{u}} \frac{1}{d_{u} d_{v}}\left(\sum_{W_{i} \notin W_{u}^{*}} \sum_{z \in W_{i}} \frac{\delta^{1 / 4} d_{u} e(z, v)}{\operatorname{vol}(G)}\right)^{2} \\
= & \sqrt{\delta} \sum_{u} \sum_{v \notin X_{u}} \frac{1}{d_{u} d_{v}}\left(\sum_{W_{i} \notin W_{u}^{*}} \frac{d_{u} e\left(W_{i}, v\right)}{\operatorname{vol}(G)}\right)^{2} \\
\leq & \sqrt{\delta} \sum_{u} \sum_{v \notin X_{u}} \frac{1}{d_{u} d_{v}}\left(\sum_{W_{i} \notin W_{u}^{*}} \frac{d_{u}}{\operatorname{vol}(G)}(1+\sqrt{\delta}) \frac{d_{v} \operatorname{vol}\left(W_{i}\right)}{\operatorname{vol}(G)}\right)^{2} \quad \text { by def. of } X_{u} \\
\leq & \sqrt{\delta} \sum_{u} \sum_{v \notin X_{u}} \frac{1}{d_{u} d_{v}}\left((1+\sqrt{\delta}) \frac{d_{u} d_{v}}{\operatorname{vol}(G)}\right)^{2} \\
\leq & \sqrt{\delta}+3 \delta
\end{aligned}
$$

(upper bounded by the sum over all $u$ and $v$ ).

Finally, for the first sum we have

$$
\begin{aligned}
& \sum_{u} \sum_{v \notin X_{u}} \frac{1}{d_{u} d_{v}}\left(\sum_{W_{i} \notin W_{u}^{*}} \sum_{z \in W_{i}} \frac{i \delta^{1 / 4} d_{u} e(z, v)}{\operatorname{vol}(G)}-\frac{d_{u} d_{v}}{\operatorname{vol}(G)}\right)^{2} \\
&= \sum_{u} \sum_{v \notin X_{u}} \frac{1}{d_{u} d_{v}}\left(\sum_{W_{i} \notin W_{u}^{*}} \frac{i \delta^{1 / 4} d_{u} e\left(W_{i}, v\right)}{\operatorname{vol}(G)}-\frac{d_{u} d_{v}}{\operatorname{vol}(G)}\right)^{2} \\
& \leq \sum_{u} \sum_{v \notin X_{u}} \frac{2}{d_{u} d_{v}}\left(\left(\sum_{W_{i} \notin W_{u}^{*}} \frac{i \delta^{1 / 4} d_{u} d_{v} \operatorname{vol}\left(W_{i}\right)}{\operatorname{vol}(G)^{2}}-\frac{d_{u} d_{v}}{\operatorname{vol}(G)}\right)^{2}\right. \\
&\left.\left.+\sum_{W_{i} \notin W_{u}^{*}} \frac{i \delta^{1 / 4} d_{u} d_{v} \operatorname{vol}\left(W_{i}\right)}{\operatorname{vol}(G)^{2}}\right)^{2}\right)^{2} \operatorname{by~the~def.~of~} X_{u}, \\
& \leq \sum_{u} \sum_{v \notin X_{u}} \frac{2}{d_{u} d_{v}}\left(2\left(\sum_{i} \frac{i \delta^{1 / 4} d_{u} d_{v} \operatorname{vol}\left(W_{i}\right)}{\operatorname{vol}(G)^{2}}-\frac{d_{u} d_{v}}{\operatorname{vol}(G)}\right)^{2}\right. \\
&\left.+2\left(\frac{\sum_{W_{i} \subseteq W_{u}^{*}} i \delta^{1 / 4} d_{u} d_{v} \operatorname{vol}\left(W_{i}\right)}{\operatorname{vol}(G)^{2}}\right)^{2}\left(\sum_{W_{i} \notin W_{u}^{*}} \frac{i \delta^{1 / 4} d_{u} d_{v} \operatorname{vol}\left(W_{i}\right)}{\operatorname{vol}(G)^{2}} \sqrt{\delta}\right)^{2}\right)^{2} \\
& \leq \sum_{u, v} \frac{1}{d_{u} d_{v}}\left(4\left(\frac{d_{u} d_{v} \delta^{1 / 4}}{\operatorname{vol}(G)}\right)^{2}+4\left(\frac{C^{\prime 2} \delta^{1 / 4} d_{u} d_{v} \delta^{1 / 2}}{\operatorname{vol}(G)}+2\left(\frac{C^{\prime} d_{u} d_{v} \delta^{3 / 4}}{\operatorname{vol}(G)}\right)^{2}\right)\right. \\
& \text { by }(8), \operatorname{def} . \text { of } W_{u}^{*} \text { and the fact that } i<C^{\prime}, \\
& \leq 4 \sqrt{\delta}+4 C^{\prime 4} \delta^{3 / 2}+2 C^{\prime 2} \delta^{3 / 2} .
\end{aligned}
$$

Now, we have to put everything together.

Random Structures and Algorithms DOI 10.1002/rsa 
First observe that

$$
\begin{aligned}
& \sum_{u, v \in V} \frac{1}{d_{u} d_{v}}\left(e_{t}(u, v)-\frac{d_{u} d_{v}}{\operatorname{vol}(G)}\right)^{2} \\
= & \sum_{u, v \in V} \frac{1}{d_{u} d_{v}} e_{t}(u, v)^{2}-2 \sum_{u, v \in V} \frac{e_{t}(u, v)}{\operatorname{vol}(G)}+\sum_{u, v \in V} \frac{d_{u} d_{v}}{\operatorname{vol}(G)^{2}} \\
= & \sum_{C_{2 t} t^{2 t-\text { circuit }}} w\left(C_{2 t}\right)-2 \frac{e_{t}(V, V)}{\operatorname{vol}(G)}+1 \\
= & \sum_{C_{2 t} t^{2 t-\text { circuit }}} w\left(C_{2 t}\right)-1
\end{aligned}
$$

(using $e_{t}(V, V)=\operatorname{vol}(G)$ ) so that the preceding results, including inequalities (11), (12), (13) and (14), give

$$
\begin{aligned}
& \left|\sum_{C_{2 t} 2 t \text {-circuit }} w\left(C_{2 t}\right)-1\right| \\
= & \sum_{u, v \in V} \frac{1}{d_{u} d_{v}}\left(e_{t}(u, v)-\frac{d_{u} d_{v}}{\operatorname{vol}(G)}\right)^{2} \\
\leq & 2 C^{\prime} C^{2} \varepsilon / \delta+2 C^{2}\left(C^{\prime}+1\right)^{2} \delta+4(\sqrt{\delta}+3 \delta)+4\left(4 \sqrt{\delta}+4 C^{\prime 4} \delta^{3 / 2}+2 C^{\prime 2} \delta^{3 / 2}\right) \\
\leq & 2 C^{\prime} C^{2} \varepsilon / \delta+2 C^{2}\left(C^{\prime}+1\right)^{2} \delta+20 \sqrt{\delta}+12 \delta+16 C^{\prime 4} \delta^{3 / 2}+8 C^{\prime} 2 \delta^{3 / 2} .
\end{aligned}
$$

This proves Theorem 3 .

Corollary 1. If $G$ has minimum degree $\alpha$, then

$$
\operatorname{DISC}(\varepsilon) \Longrightarrow \operatorname{CIRCUIT}_{2 t}(\eta)
$$

where $\eta$ depends only on $\varepsilon, \alpha$ and $t$.

Theorem 4. If $G$ has minimum degree $\alpha$ for some constant $\alpha$, then $\mathrm{CIRCUIT}_{2 t}, \mathrm{TRACE}_{2 t}$, EIG, DISC, DISC $_{2}$, DISC $_{t}$ are all equivalent for $t \geq 2$.

\section{CONCLUDING REMARKS}

We can summarize the main theorems in the following:

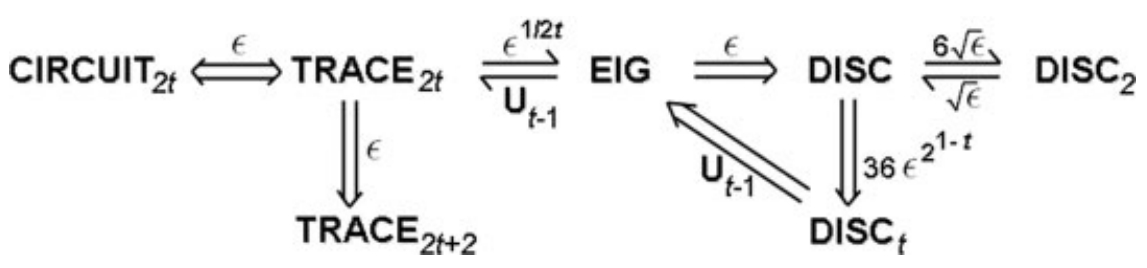

Fig. 2. Quasi-random properties for $G_{n}(\mathbf{d})$. 
We should note that if for our degree sequence $\mathbf{d}$, we choose all $d_{i}$ to be (approximately) equal, so that the $G \in \mathcal{G}(\mathbf{d})$ are (approximately) regular, then our results specialize to the case of sparse random graphs considered in [20], except that here we get explicit functions of $\varepsilon$ (as opposed to the expressions with $o(1)$ terms occurring in [20]). What are other properties which might be included in Theorem 1? Can condition $\mathrm{U}_{t-1}$ be replaced by a weaker condition to allow DISC $\Rightarrow \mathrm{CIRCUIT}_{2 t}$ to be proved (Fig. 2)? We hope to return to this in the future.

\section{ACKNOWLEDGEMENTS}

The authors thank Steven Butler for careful readings of the paper. His many comments made substantial improvements to the proofs.

\section{REFERENCES}

[1] W. Aiello, F. Chung, and L. Lu, "A random graph model for massive graphs," In Proceedings of the 32nd Annual ACM Symposium on Theory of Computing, New York, 2000, pp. 171-180.

[2] W. Aiello, F. Chung, and L. Lu, A random graph model for power law graphs, Exp Math 10 (2001), 53-66.

[3] W. Aiello, F. Chung, and L. Lu, "Random evolution in massive graphs," In Eds. J. Abello, et al., Massive Comput. 4, Kluwer Academic Publishers, 2002, pp. 97-122.

[4] N. Alon and J. H. Spencer, The probabilistic method, Wiley, New York, 1992.

[5] A.-L. Barabási and R. Albert, Emergence of scaling in random networks, Science 286 (1999), 509-512.

[6] A.-L. Barabási, R. Albert, and H. Jeong, Scale-free characteristics of random networks: The topology of the world wide web, Physica a 281 (2000), 69-77.

[7] Y. Bilu and N. Linial, Lifts, discrepancy and nearly optimal spectral gap (in press).

[8] B. Bollobás and V. Nikiforov, Hermitian matrices and graphs: Singular values and discrepancy, Discrete Math 285 (2004), 17-32.

[9] A. Broder, R. Kumar, F. Maghoul, P. Raghavan, S. Rajagopalan, R. Stata, A. Tompkins, and J. Wiener, "Graph Structure in the Web," In Proceedings of the WWW9 Conference, May, Amsterdam, 2000.

[10] S. Butler, Using descrepancy to control singular values for nonnegative matrices, Linear Algebra Appl 419 (2006), 486-493.

[11] F. Chung, Spectral graph theory, AMS Publications, 1997, xii+207 pp.

[12] F. R. K. Chung, The regularity lemma for hypergraphs and quasi-randomness, Random Struct Algorithms 2 (1991), 241-252.

[13] F. R. K. Chung and R. L. Graham, Cohomological aspects of hypergraphs, Trans Am Math Soc 334 (1992), 365-388.

[14] F. R. K. Chung and R. L. Graham, "Maximum cuts and quasi-random graphs," In Eds. Alan Frieze and Tomasz Luczak, Random graphs, Wiley, New York, 1992, pp. 23-34.

[15] F. R. K. Chung and R. L. Graham, On graphs not containing prescribed induced subgraphs, In Eds. A. Baker, et al., A tribute to Paul Erdö, Cambridge University Press, 1990, pp. 111-120.

[16] F. R. K. Chung and R. L. Graham, Quasi-random hypergraphs, Random Struct Algorithms 1 (1990), 105-124. 
[17] F. R. K. Chung and R. L. Graham, Quasi-random set systems, J Am Math Soc 4 (1991), 151-196.

[18] F. R. K. Chung and R. L. Graham, Quasi-random subsets of $Z_{n}$, J Combin Theory (A) 61 (1992), 64-86.

[19] F. R. K. Chung and R. L. Graham, Quasi-random tournaments, J Graph Theory 15 (1991), 173-198.

[20] F. Chung and R. L. Graham, Sparse quasi-random graphs, Combinatorica 22 (2002), 217-244.

[21] F. R. K. Chung, R. L. Graham, and R. M. Wilson, Quasi-random graphs, Combinatorica 9 (1989), 345-362.

[22] F. Chung, L. Lu, T. G. Dewey, and D. J. Galas, Duplication models for biological networks, J Comput Biol 10 (2003), 677-688.

[23] F. Chung, L. Lu, and V. Vu, The spectra of random graphs with given expected degrees, Proc Nat Acad Sci USA 100 (2003), 6313-6318.

[24] C. Cooper and A. Frieze, A general model of undirected Web graphs, Random Struct Algorithms 22 (2003), 311-335.

[25] J. Cooper, Quasirandom permutations, J Comb Theory A 106 (2004), 123-143.

[26] M. Faloutsos, P. Faloutsos, and C. Faloutsos, On power-law relationships of the Internet topology, SIGCOMM'99 29 (1999), 251-262.

[27] J. Grossman, P. Ion, and R. de Castro, Facts about Erdös numbers and the collaboration graph, http://www.oakland.edu/ grossman/trivia.html.

[28] H. Jeong, B. Tomber, R. Albert, Z. Oltvai, and A. L. Babárasi, The large-scale organization of metabolic networks, Nature 407 (2000), 378-382.

[29] J. Kleinberg, S. R. Kumar, P. Raphavan, S. Rajagopalan, and A. Tomkins, The web as a graph: Measurements, models and methods, In Proceedings of the International Conference on Combinatorics and Computing, Tokyo, 1999.

[30] M. Krivelevich and B. Sudakov, "Pseudo-random graphs," More sets, graphs and numbers, E. Gyori, G. O. H. Katona, and L. Lovász (Editors), Bolyai Soc. Math. Stud. 15, Springer, Berlin, 2006, pp. 199-266.

[31] A. Thomason, "Pseudo-random graphs," In Ed. M. Karónski, Ann Discrete Math 33 (1987), 307-331. Proceedings of Random Graphs, Poznán.

[32] A. Thomason, Random graphs, strongly regular graphs and pseudo-random graphs, In Ed. C. Whitehead, Survey in combinatorics, 1987; London Math Soc Lecture Note Ser 123 (1987), 173-195.

[33] D. West, Introduction to graph theory, Prentice Hall, Upper Saddle River, NJ, 1996, xvi+512 p. 